\title{
Compacidad y densidad de las ciudades españolas
}

Fernando García. Universidad Politécnica de Cartagena, Cartagena, España.

RESUMEN | Las características espaciales de las ciudades resultan decisivas en los principales problemas urbanos: la calidad de vida, la movilidad, el consumo energético y de materiales, etcétera. Para describir el crecimiento de las ciudades, la compacidad y la densidad se han convertido en dos de los valores más funcionales, y pueden ser calculados a través de las principales bases de datos de coberturas del suelo en España: CORINe Land Cover y siose. El análisis de los rangos de estos valores para distintos tipos de ciudades permite caracterizar la forma de las ciudades españolas, comparando sus diferencias. A partir de esta definición espacial, se abre la posibilidad de estudiar el efecto de la forma de la ciudad en los distintos problemas y retos a los que se enfrenta la sociedad urbana.

PALABRAS CLAVE | morfología urbana, expansión urbana, indicadores ambientales.

ABSTRACT | The spatial characteristics of cities are crucial in major city problems, including quality of life, mobility, energy and material consumption, etc. To describe the cities sprawl, compactness and density have become two of the most functional values, and can be obtained through the main land cover databases in Spain: CORINE Land Cover and SIOSE. The analysis of the ranges of these values for different types of cities allows us to characterize the form of the Spanish cities and compare their differences. This spatial definition opens the possibility of studying the effect of the shape of the city in the various problems and challenges facing urban society.

KEYWORDS | urban morphology, urban sprawl, environmental indicators. 


\section{Introducción}

El crecimiento de la población urbana en España desde inicios del siglo xx ha sido espectacular. A principios del siglo pasado, apenas un tercio de la población vivía en núcleos de más de 10.000 habitantes, un porcentaje que se ha incrementado hasta el 80\% según el último Censo de población, correspondiente al año 2011. Son 110 años en los que la base rural de la estructura demográfica ha dado paso a una sociedad primordialmente urbana, en la que más de la mitad de la población se concentra en ciudades mayores a los 50.000 habitantes, frente a la sexta parte que lo hacía en 1900.

Este proceso de urbanización es un fenómeno global. Según Naciones Unidas (Bellet \& Llop, 1999), en 1950 el 29\% de la población del planeta, unos 734 millones de personas, vivía en ciudades; y en 1994, este porcentaje llegó a un 45\%, 2.500 millones. A pesar de que el cálculo de estas cifras es frágil, se considera que entre $40 \%$ y $55 \%$ de la población actual vive en asentamientos urbanos y la previsión es que en las primeras décadas de este siglo se alcance el $60 \%$ de población en esas condiciones. Estos valores se ven notablemente aumentados si nos centramos en un entorno más reducido y próximo, como la Unión Europea, en el que se calcula que el $68 \%$ de la población vive ya en entornos urbanos (European Commission, 2011).

El crecimiento de las ciudades ha estado acompañado por fuertes cambios en sus características espaciales, un proceso tendiente a la configuración de un modelo cada vez más disperso del territorio. En el estudio a nivel mundial de Angel, Parent, Civco y Blei (2011) se detectó que el crecimiento de la población en el siglo xx fue del 1,6\% anual, mientras que el aumento del uso del suelo fue del 3,66\%, produciendo una caída general de la densidad. Las ciudades europeas, si bien mantuvieron su histórica compacidad hasta mitad del siglo $\mathrm{xx}$, a partir de entonces se expandieron una media de $78 \%$, pese a que la población solo aumentó un 33\%. Además, se ha observado que se trata de un fenómeno generalizado, de parecida intensidad independientemente de la densidad de población previa de cada ciudad y que incluso se mantiene en las zonas donde la población desciende (European Environment Agency, 2006).

En España, las superficies artificiales se estiman en un 1\% (Angel et al., 2011), distribuidas de forma irregular en el territorio con una concentración dominante en el área metropolitana de Madrid y en el anillo litoral, que se hace mucho más intensa en el arco mediterráneo. La evolución de la morfología de las ciudades ejemplifica el cambio de modelo urbano, que, a pesar de las características locales de cada caso, puede generalizarse en fases descritas ya en varias ocasiones (Capel, 1975; Terán, 1982; Azcárate et al., 2010). Hasta la mitad del siglo xix, el crecimiento urbano se realizó en continuidad con el centro histórico. A partir de ese momento, las ciudades iniciaron su industrialización, surgiendo, en muchos casos,

1 Este artículo presenta contenidos desarrollados como parte de la tesis en curso del autor, dirigida por el profesor Luis Moya González (Departamento Urbanística y Ordenación del Territorio de la Universidad Politécnica de Madrid [upm]) dentro del Programa de Doctorado Periferias, Sostenibilidad y Vitalidad Urbana de la UPM. 
una pequeña proporción de núcleos obreros de baja densidad más o menos periféricos. Pero la verdadera explosión en superficie no llegaría hasta las décadas de los sesenta y setenta del siglo $\mathrm{xx}$, con una economía favorable y una fuerte inmigración rural. Estos años fueron el primer periodo de dispersión de las ciudades españolas, que se vería frenado en los ochenta por la crisis económica, lo que permitió incluso emprender operaciones de cosido de los tejidos inconexos (Moya, 1983). El cambio de siglo trajo el segundo periodo de dispersión, dominado por dos modelos: una periferia urbana que padecía de un sobredimensionamiento del espacio público, y una periferia suburbana de baja densidad que se aprovechaba de la gran expansión de las infraestructuras (López de Lucio, 2007; Farińa \& Naredo, 2010).

A este reciente modelo urbano disperso se le ha diagnosticado una serie de problemas que afectan la sostenibilidad general del planeta: elevado impacto ambiental, segregación social, incremento de los desplazamientos, ineficiencia económica, etcétera. Por ello, los organismos internacionales han establecido el objetivo de frenar la dispersión, potenciando los modelos de ciudad compacta con menos consumo de suelo, propósito incluido explícitamente en la Carta de Aalborg de 1994, la Declaración de Hannover del 2000, la Visión Aalborg + 10 de 2004 y la Carta de Leipzig de 2007.

En la resolución de estos problemas, las características espaciales de la ciudad resultan clave, siendo la densidad el valor más empleado. Esta mide habitualmente la cantidad de población o de superficie construida por unidad de superficie, por lo que en su cálculo es necesario conocer también la extensión de la superficie urbana, otra de las preocupaciones que plantean los nuevos modelos urbanos. Así, la densidad se ha utilizado como referente para el estudio de la movilidad y la accesibilidad (Masnavi, 2000; Navarro \& Ortuño, 2011; Webster, 2010;), de la calidad de vida (Burton, 2000; Font, 2007; García, 2012; Uytenhaak, 2008), del consumo energético y la sostenibilidad de las ciudades (Navarro \& Ortuño, 2011; Owens, 1986; Thinh, 2002; Williams, 2000), etcétera.

Sin embargo, y tal como ya se venía observando en la arquitectura desde los años treinta (Gropious, 1930), la densidad es insuficiente para determinar la configuración del espacio urbano. Por ello, paulatinamente se ha ido incorporando una variable que permite evaluar la apertura del espacio libre o, lo que es equivalente, la compacidad de la distribución del espacio construido. Este valor es de carácter puramente espacial, y mide la relación entre la superficie ocupada y la total. Si para la arquitectura la compacidad permitía establecer relaciones claras con las tipologías arquitectónicas (Berghauser \& Pont, 2009; Kickert, Berghauser \& Nefs, 2014; Martin \& March, 1975; Steadman, 2014), en la escala de ciudad la compacidad, definida de formas diversas, ha servido para evaluar propiedades de la configuración espacial como la proximidad, la centralidad o la fragmentación (Angel et al., 2010; Farińa \& Naredo, 2010; Goerlich \& Cantarino, 2012; López de Lucio, 2007). Se trata de valores que no solo reflejan las características formales, sino que están intrínsecamente ligados al modo de vida que se desarrolla en las ciudades (Goerlich \& Cantarino, 2012). La densidad y la compacidad son indicadores de la intensidad y la concentración de actividades, aspectos esenciales en el modelo urbano disperso. 
Este artículo muestra una metodología para el cálculo de estos valores en el caso español, señalando sus limitaciones. Posteriormente se realiza una lectura de los resultados por separado y en conjunto, caracterizando la densidad y la compacidad de las ciudades españolas.

\section{Metodología}

Aunque los estudios existentes permiten cuantificar los usos del suelo, junto con las densidades y, con menor frecuencia, la compacidad, estos valores no son empleados de forma conjunta para la comprensión de las características espaciales de las ciudades. A la inversa, la metodología aplicada en el estudio que aquí se recoge permite obtener los valores pertinentes de manera que puedan combinarse posteriormente, para lo cual se emplean bases de datos de coberturas de suelo y de población ya existentes. Los proyectos gubernamentales a los que corresponden estas bases de datos de acceso libre tenían el propósito de actualizar la información periódicamente para crear series temporales. No obstante que el cumplimiento de esos objetivos está siendo irregular, el uso de estas fuentes deja abierta la posibilidad de realizar un seguimiento temporal de los valores.

El primero de los parámetros buscados es la densidad, definida habitualmente como el cociente entre un numerador referido principalmente a viviendas, habitantes o superficie de suelo construida, y un denominador que, en la mayor parte de las ocasiones, es la superficie del terreno que se analiza (Boyko \& Cooper, 2011). En este caso, se empleará la densidad de población bruta en el área urbana, es decir, el número de habitantes por unidad de superficie.

La compacidad, el segundo de los parámetros empleados, se define en este trabajo como el porcentaje de superficie del terreno ocupada por la edificación. La compacidad puede también considerarse como un tipo de densidad que expresa la relación entre el espacio construido y el no construido. ${ }^{2}$

Para definir estos valores ha sido necesario obtener la extensión de la superficie urbana, el número de habitantes y la superficie ocupada por la edificación en esa superficie.

El ámbito de estudio se compone de los municipios calificados como urbanos en la estadística española, vale decir, aquellas entidades de población con más de 10.000 habitantes (Goerlich \& Cantarino, 2013). Estos municipios albergan 37,1 millones de habitantes, el 79\% de los 46,8 millones de la población nacional. Tal separación entre lo urbano y lo rural se ajusta a las características propias de las fuentes de datos empleadas, como se verá más adelante. de sostenibilidad de Sevilla (2007) y Vitoria-Gasteiz (2009). En estos planes se ha denominado compacidad corregida al volumen edificado por espacio público de estancia, pretendiendo obtener así información sobre la intensidad de uso de los espacios libres. 


\section{Características de las fuentes de datos empleadas}

El estudio de la densidad y compacidad de las ciudades españolas es posible gracias a la existencia de bases de datos de coberturas del suelo. Distintos proyectos europeos y españoles se han encargado, a lo largo de los últimos 25 años, de cartografiar y clasificar los distintos usos del territorio a una escala suficientemente detallada para arrojar información sobre las ciudades. La accesibilidad a estos datos a través de los portales web de los distintos organismos y el desarrollo de las aplicaciones informáticas conocidas como Sistemas de Información Geográfica permiten emplear los resultados de estos proyectos con nuevos fines.

En esta investigación se han utilizado los datos del Coordination of Information on the Environment - Land Cover (CLC o CORINe Land Cover) y del Sistema de Información sobre Ocupación del Suelo de España (sIOsE). También se empleó el grid, o rejilla, de población realizado por el proyecto GEOSTAT, financiado por Eurostat, la oficina europea de estadística.

El programa CORINE se estableció en 1985 por la Comisión Europea (CE/338/85) con el objetivo de recopilar, coordinar y homogeneizar la información sobre el estado del medioambiente y los recursos naturales a nivel europeo (Goerlich \& Cantarino, 20I3). En el marco de este programa, el proyecto CLC se ha ocupado de crear una base de datos europea sobre usos (o coberturas) del territorio mediante la interpretación de las imágenes recogidas por los satélites Landsat y Spot 33. En la actualidad, el proyecto es responsabilidad de la Agencia Europea de Medio Ambiente (AEmA; European Environment Agency, EEA) y forma parte del Land Core Monitoring System de Gmes (Global Monitoring for Environment and Security) (Hurtado, 2013). Hay datos publicados para 1990, 2000 y 2006 como fechas de referencia, y está pendiente de publicación la edición de 2012.

Por su parte, el programa siose tiene como objetivo integrar la información de las bases de datos de coberturas y usos del suelo de las distintas Comunidades Autónomas y de la Administración General del Estado Español (www.siose.es). El proyecto se gestó durante la participación española en el CLC de 1990 y fue actualizado en el año 2000 (Goerlich, 2013), ya que en la realización del siose se detectó la necesidad de aumentar la escala y coordinar las diferentes iniciativas de las Comunidades Autónomas. En octubre de 2005 se firmó un protocolo entre los Ministerios de Defensa, Fomento y Medio Ambiente para la obtención de coberturas del territorio español a través de imágenes satelitales, en el marco del Plan Nacional de Teledetección.

La Dirección General del Instituto Geográfico Nacional (IGN), del Ministerio de Fomento, junto con la Secretaría General para el Territorio y la Biodiversidad del Ministerio de Medio Ambiente, coordinó el proyecto apoyándose en la European Environment Information and Observation Network (EIONET). Existen tres versiones, con fechas de referencia de los año 2005, 2009 y 2011 (estas dos últimas accesibles para todo el territorio nacional únicamente desde marzo de 2015).

La comparación de ambos proyectos muestra las características propias de cada uno. En primer lugar, debe asumirse el distinto ámbito al que se refieren, ocupándose ClC de la totalidad de la Comunidad Europea y siose del territorio del Estado español. Comparten, sin embargo, características técnicas, como el sistema geodésico 
de referencia ETRS89 y una proyección UTM (Universal Transverse Mercator), con huso 30 extendido para CLC y 28, 29, 30 y 31 en siose, según el correspondiente a cada Comunidad Autónoma.

Una diferencia fundamental entre ambos proyectos es la escala, mucho más detallada la de siose, 1:25.000, que la de CLC, 1:100.000. Esta diferencia queda reflejada en la unidad mínima cartografiable de cada proyecto, es decir, la dimensión mínima que debe tener un área para ser delimitada como una cobertura independiente. En ClC esta unidad mínima, MMU (Minimum Mapping Unit) es de 25 hectáreas, mientras que en siose varía según el tipo de cobertura: 0,5 ha para cultivos forzados, coberturas húmedas, playas, vegetación de ribera y acantilados marinos; 1 ha para zonas urbanas y láminas de agua; y 2 ha para zonas agrícolas, forestales y naturales. Además, dada la singularidad de los elementos lineales, ambos proyectos establecen un ancho mínimo del elemento que será cartografiado, siendo de 100 m en CLC y de $15 \mathrm{~m}$ en SIOSE.

La segunda gran diferencia entre ambos proyectos está en la estructura de la información de coberturas empleada. En CLC se utiliza un modelo jerárquico en el que a cada porción del territorio relativamente homogénea se le asigna una cobertura única, correspondiente a su uso mayoritario, de entre las incluidas en una clasificación de 3 niveles y 44 clases en su máximo nivel de desagregación (Bossard, Feranec \& Otahel, 2000; Büttner, Feranec \& Jaffrain, 2006). El siose, por su parte, se estructura en un modelo de datos con filosofía de orientación a objetos (Goerlich \& Cantarino, 2013), en el que el objetivo no es clasificar cada porción del terreno, sino describirla. Existe una clasificación de 40 coberturas simples que se combinan de diferentes formas, constituyendo coberturas denominadas "compuestas". Estas combinaciones pueden ser únicas de cada objeto, de acuerdo con el porcentaje de superficie de cada una de las coberturas simples, o predefinidas. Las coberturas compuestas predefinidas representan combinaciones de coberturas simples representativas dentro del territorio, como, por ejemplo, el tejido urbano de ensanche. En SIOSE quedan recogidas todas las coberturas que representan más de un $5 \%$ de una porción del territorio.

La combinación de la menor escala de trabajo y el modelo de estructura de la información supone un aumento notable de la resolución de siose respecto a CLC. Y, para los objetivos de este trabajo, destaca el detalle con que describe las coberturas artificiales, puesto que 41 de las 45 coberturas compuestas predefinidas corresponden a este tipo, según sea su estructura urbana, la actividad económica, los equipamientos o las infraestructuras. Sin duda, la integración de fuentes de datos correspondientes al planeamiento urbano y territorial colaboró fundamentalmente en este nivel de detalle.

Como contraste, el modelo jerárquico del cLc es más difícil de ajustar a la realidad del territorio, pues no siempre es posible encontrar una cobertura dominante en un polígono, en cuyo caso este se clasifica como heterogéneo. Esto sucede en el 17,8\% del territorio español (Goerlich \& Cantarino, 2013) y es también parte de la causa por la que la superficie artificial se reduce al $2 \%$. Sin embargo, como se verá, las características de ambos proyectos son apropiadas para los objetivos de este estudio. 
FIGURA I | Comparación de la resolución geométrica entre sIOsE 2005 y CLC 2006

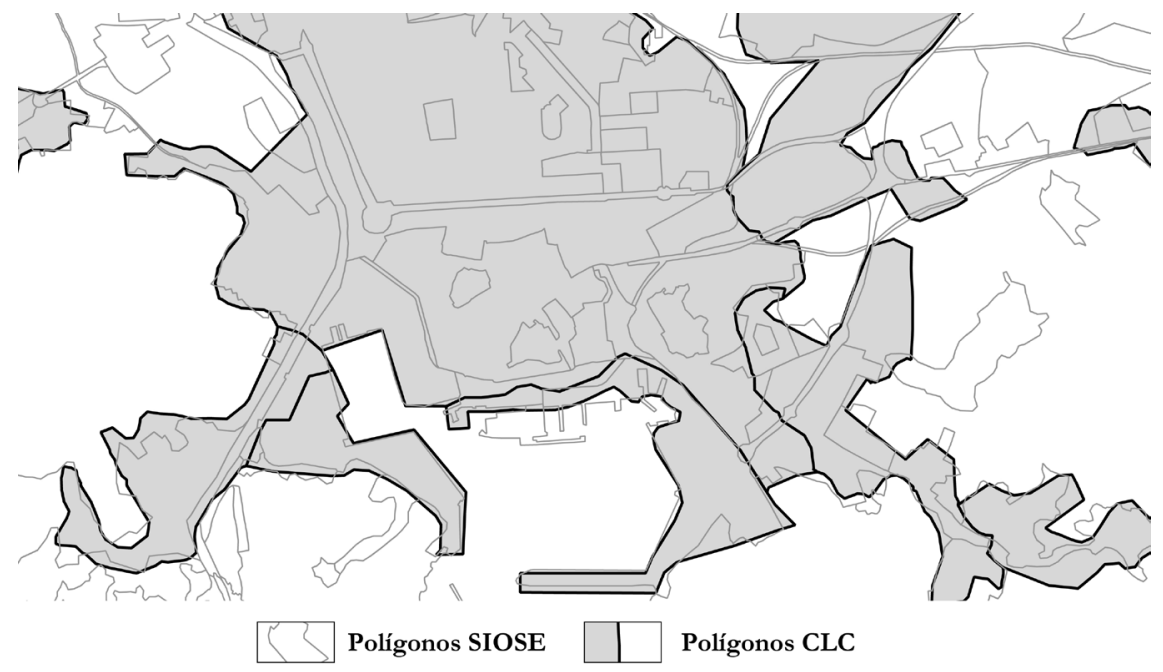

FUENTE GOERLICH \& CANTARINO, 2013

La tercera de las fuentes de datos empleadas no es una base de datos de coberturas del suelo, como las anteriores, sino de distribución de la población sobre el territorio. El proyecto GeOstat fue iniciado por el European Forum for GeoStatistics (EFGS) para promover un sistema estadístico basado en una rejilla (grid), con el objetivo más genérico de integrar la información estadística en un sistema geoespacial común. Varios miembros del EFGs trabajaron en un primer prototipo (GEOSTAT 1A) con los datos de población del año 2006, en el que la información española se extrajo de los trabajos de Goerlich y Cantarino (2012). Posteriormente, con los datos de los Censos del año 2011, se ha creado una primera versión (GEOstaT Iв), que incluye los datos oficiales elaborados por las oficinas de estadística de 18 de los países de la Unión Europea y la Asociación Europea de Libre Comercio (EFTA, por sus siglas en inglés), entre los que se sitúa España, y datos de otros 14 países obtenidos por modelización. Una nueva versión del proyecto (GEOSTAT IC) estaba prevista para el año 2015, pero no ha sido publicada.

En España, la georreferenciación de los datos del Censo de 2011 ha sido una novedad respecto a censos anteriores y ha permitido elaborar la información para GEOSTAT por agregación de los datos individuales. La rejilla así obtenida es más precisa que la habitual división en secciones censales en los límites de las áreas urbanas, donde estas se extienden en superficies mayores a $1 \mathrm{Km}^{2}$ hasta que alcanzan volumen mínimo de población.

Dado que las bases de datos no coinciden en los años a los que se refieren, se han empleado las versiones más actuales y más próximas en el tiempo entre sí: siosE 2005, ClC 2006 y geostat i в 2011. 


\section{Superficie de referencia o de cálculo}

Para delimitar la superficie de referencia en la que se calcularán los parámetros, se ha empleado aquella calificada como artificial por el proyecto CLC en cada municipio, evitando así las distorsiones habituales ocasionadas al emplear delimitaciones administrativas (Goerlich \& Cantarino, 2013).

Es necesario destacar que la unidad mínima cartografiable de esta fuente de datos, 25 hectáreas, impone algunas limitaciones al estudio, ya que edificaciones y núcleos que no forman conjuntos de la extensión suficiente no son delimitados como artificiales. Por ello existen 4.199 términos municipales, de los 8.118 totales, en los que ClC no delimita ninguna superficie artificial, sus núcleos no son pues lo suficientemente extensos para ser cartografiados. Analizando estos casos se observa que se trata de municipios de menos de 1.000 habitantes en el Censo de 2011 (solo cuatro de ellos tienen más de 5.000 y solo uno de ellos más de 10.000). Dado que este trabajo se ciñe a los 755 municipios con más de 10.000 habitantes en el Censo de 2011 - los considerados como urbanos en la estadística oficial española—, esta carencia de datos no tiene relevancia.

Por esta misma razón, el estudio se centra en los cascos urbanos principales de cada municipio, descartando en muchos casos crecimientos periféricos aislados o muy dispersos que no alcanzan la superficie mínima cartografiable. El estudio, por lo tanto, obtendrá resultados en la definición de las características espaciales de los entornos que podríamos denominar "puramente urbanos", las cuales permiten una valoración de la intensidad y la concentración de usos de los ciudadanos, pero no son útiles para conocer la estructura funcional del territorio en que se insertan.

\section{Superficie ocupada por la edificación}

Se calcula la superficie ocupada utilizando las coberturas correspondientes a edificación (código 101), delimitadas por el proyecto siose. Para el cálculo de este valor se intersectan, en primer lugar, los polígonos del siose con la superficie de referencia. A continuación se recalcula su área y la superficie ocupada por la edificación, empleando los porcentajes correspondientes a cada tipo de cobertura indicados en la descripción de los polígonos.

La información del SIOSE también habría permitido atribuir la superficie ocupada a distintos tipos de edificación —edificación aislada (código 21), edificación entre medianeras (22), vivienda unifamiliar aislada (23), vivienda unifamiliar adosada (24) y nave (25) — , pero no se ha empleado esta información, a fin de evitar complicar la posterior lectura de los resultados.

\section{Número de habitantes}

En el cálculo de la densidad de población es necesario realizar algunas operaciones con la rejilla de población GeOstat i в de 2011, tanto de agregación como de desagregación, por lo que se genera un factor de error. En primer lugar se aíslan todas las celdas que coinciden con la superficie de cálculo de cada municipio. Hecha esta selección, se presentan tres tipos de situaciones básicas, que pueden observarse en la figura 2: que la celda esté completamente en la superficie artificial de un municipio 
(celda A), que esté parcialmente sobre dicha superficie (B), y que la celda esté sobre dos o más municipios distintos $(\mathrm{C})$.

En las dos primeras situaciones, A y B, con la celda situada sobre un solo municipio, se ha asignado toda la población de la celda a ese municipio. En el caso B, en que la celda está parcialmente sobre la superficie de cálculo, este criterio supone un margen de error, dado que parte de la población censada podría habitar fuera de las coberturas delimitadas como artificiales. No obstante, de acuerdo con las definiciones de cada tipo de superficie en CLC (Büttner et al., 2006), se deduce que en las superficies no delimitadas como artificiales la población es de muy baja densidad, lo que hace reducido el error posible.

En la tercera de las situaciones, $\mathrm{C}$, con la celda sobre varios municipios, se sigue el criterio de repartir la población de manera proporcional a la superficie artificial de cada municipio incluida en la celda. Aunque las coberturas artificiales de cLC son de diversa índole, con este criterio se les supone un grado de homogeneidad mínimo que les permita albergar una cantidad de población similar.

FIGURA 2 | Situaciones posibles de las celdas de la rejilla GEOSTARIB respecto a las superficies artificiales de CLC

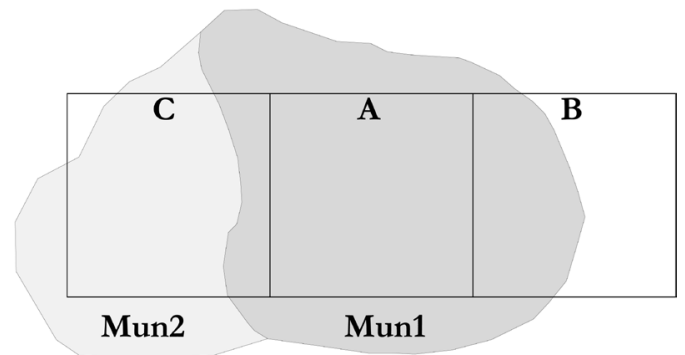

FUENTE ELABORACIÓN PROPIA

Aun teniendo en cuenta estas situaciones, al agregar la población del grid para cada municipio se dio una serie de casos (162, el 21,5\% del total) en que la población total resultante de la ciudad era superior a la censada. Además de por los posibles errores ya comentados, este hecho puede darse cuando existen núcleos de población menores a la unidad mínima cartografiable dentro de las celdas empleadas. Para evitar tal incongruencia, se asignó a estos municipios la población recogida en el censo.

\section{Compacidad y densidad en las ciudades españolas}

Los valores de la superficie de referencia, la superficie ocupada por la edificación y el número de habitantes obtenidos son, por sí mismos, útiles para conocer el grado de urbanización de un municipio. Para los objetivos de este trabajo, resultan más decisivos los índices de densidad y compacidad calculados a partir de los valores anteriores. Estos índices representan mejor las relaciones que se producen entre las tres variables iniciales, al arrojar más información sobre las características espaciales de las distintas ciudades, la proporción entre sus espacios libres y construidos y 
la intensidad de las actividades que en ellas se desarrollan. Además, densidad y compacidad permiten establecer comparaciones entre varias ciudades, al ser valores adimensionales referidos a la unidad de superficie.

Para completar la comprensión de la relación existente entre las tres variables iniciales es especialmente útil el uso de un diagrama denominado Spacemate, empleado por Berghauser y Haupt (2009) para comparar fragmentos urbanos. En el presente estudio ha sido adaptado a las particularidades propias de la escala de trabajo en que se opera, con la denominación Citymate. Este diagrama permite comparar las características espaciales de un conjunto de ciudades empleando de forma integrada los índices de compacidad y densidad.

En los epígrafes siguientes se expondrán los resultados de la investigación, comenzando con los valores de superficie artificial, para que sirvan como contexto de la dimensión de las distintas ciudades. Posteriormente se analizarán los índices de densidad y compacidad por separado, para, finalmente, comparar las características espaciales del conjunto de ciudades seleccionado a través del Citymate.

Con el fin de analizar los resultados, se han dividido las ciudades en grupos según su población en el Censo de 2011, evitando establecer comparaciones entre municipios muy distintos funcionalmente. Cualquier clasificación de tipos de ciudades resulta inevitablemente controvertida, dado que son organismos tan complejos y particulares que las agrupaciones solo pueden realizarse mediante reducciones a aspectos más generales. En la bibliografía sobre clasificación de ciudades es común indicar que la pertenencia de una ciudad a un nivel o a otro no es cuestión exclusiva de su población, sino de la relevancia funcional de esa ciudad en el conjunto y en su entorno más inmediato. No obstante, Jacobo Blanco (2004) reconoce que "al menos en Europa, la población puede considerarse sinónima de rango económico de la ciudad y reflejar su grado de actividad, sin inducir a distorsiones de importancia” (p. 126).

En esta ocasión, clasificar las ciudades según su población guarda una relación directa con las cualidades espaciales estudiadas: la extensión de la superficie artificial y la densidad, tal como se expone en los apartados correspondientes. La incorporación de otras variables a la clasificación de ciudades complejizaría este estudio innecesariamente, aunque sí sería recomendable en otras investigaciones en las que se emplee la compacidad y la densidad con otros objetivos.

La clasificación resultante se compone, en primer lugar, por el conjunto de las grandes ciudades, aquellas con población superior a 600.000 habitantes (5 casos). Madrid destaca como el municipio más poblado (3.198.645 hab.) con una amplia diferencia sobre Barcelona (1.611.013), siendo los dos únicos municipios por encima del millón de habitantes. Cabe destacar que esta fuerte diferencia de población se reduce si tenemos en cuenta las delimitaciones que realiza Aguado (2013), en las que el área urbana de Madrid alcanza 6.052.247 habitantes y la de Barcelona los 5.030.679. Junto a estas dos grandes metrópolis, Valencia, Sevilla y Zaragoza también superan los 600.000 habitantes, siendo — de nuevo según Aguado- el área urbana de las dos primeras mayor del millón y medio, mientras que la de Zaragoza apenas llega a la mitad. 
FIgURA 3 | Distribución de la población según Censo 2011 por tipos de ciudades

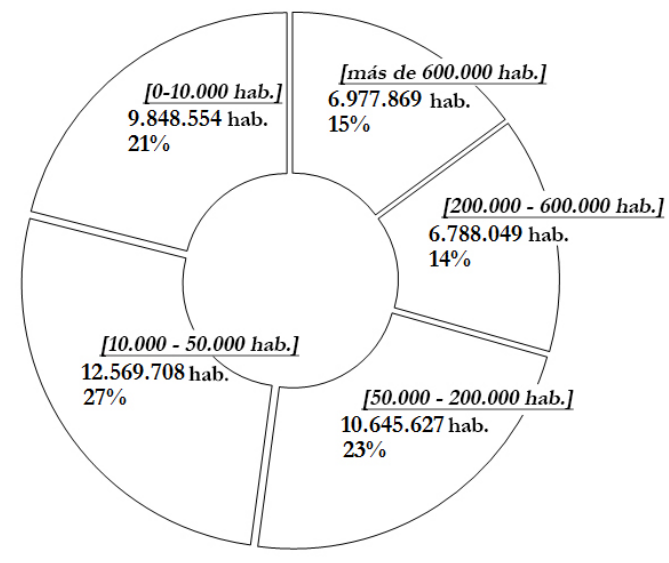

FUENTE ELABORACIÓN PROPIA

A continuación se considera el grupo de las ciudades intermedias, 24 términos municipales con entre 200.000 y 600.000 habitantes, sin superar ninguno de ellos el millón de habitantes en su área metropolitana. El conjunto alberga casi 7 millones de personas, alrededor del $15 \%$ de la población total de España y cifra similar a la de las grandes ciudades. Está compuesto por 14 capitales de provincia (todas litorales, salvo Córdoba y Valladolid), 6 ciudades de las coronas metropolitanas de Madrid y Barcelona y 4 ciudades que conforman ámbitos metropolitanas con sus respectivas capitales (Gijón, con Oviedo; Elche, con Alicante; Cartagena, con Murcia; y Jerez de la Frontera, con Cádiz).

Por debajo de este nivel se encuentran las ciudades medias, 115 municipios entre 50.000 y 200.000 habitantes en los cuales habita el 23\% de la población nacional (10,6 millones de personas). Este grupo de ciudades ha sido destacado (Bellet \& Llop, 1999; Ganau \& Vilagrasa, 2003; Vinuesa, 1989) por su papel en el sistema de ciudades nacional, al actuar como centros regionales o provinciales que permiten descongestionar las ciudades mayores y llevar los servicios propios de las urbes a su entorno más rural, así como por ser hábitats humanos más justos y saludables.

En un último escalón se sitúan las ciudades pequeñas, con una población de entre 10.000 y 50.000 habitantes, que en conjunto acumulan 12.569 .708 habitantes, un $27 \%$ del total, en 612 municipios. Al ser un grupo mucho más numeroso, sus subgrupos son más diversos.

\section{Extensión superficies artificiales}

En la evolución de las superficies artificiales en las tres mediciones de CLC (1991, 2000 y 2006) se refleja el fuerte crecimiento de las ciudades durante este periodo, tendencia que ha producido una gran transformación de las áreas urbanas en España. En el cuadro 1 se observa un incremento de 58,28\% de las superficies artificiales desde el año 1990 al 2006 en los municipios mayores de 10.000 habitantes, pasando de un total de $4.104 \mathrm{Km}^{2}$ a $6.495 \mathrm{Km}^{2}$. 


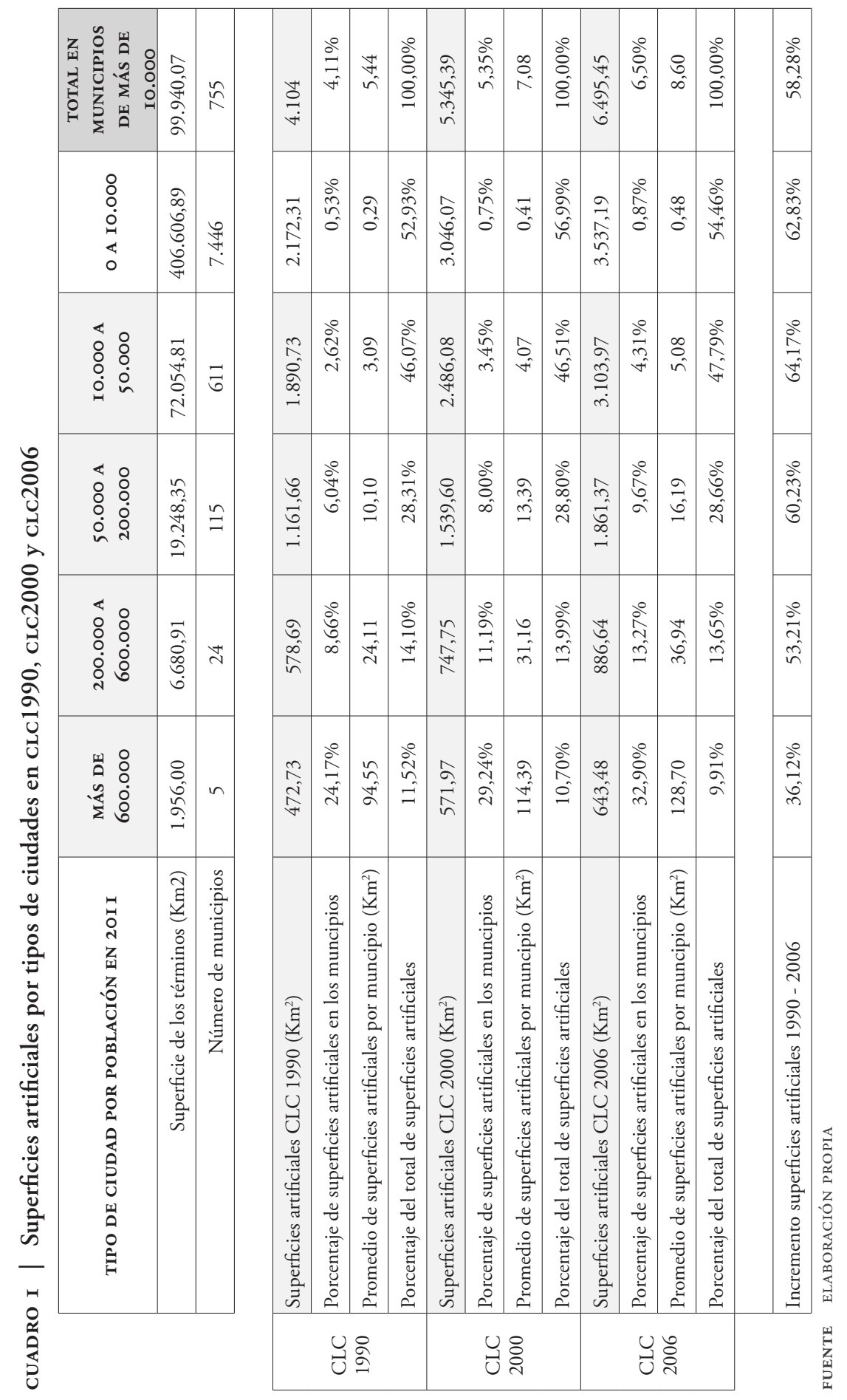


Pero si se observan los diferentes tipos de ciudades por separado, se obtienen datos sobre la distinta realidad espacial que las caracteriza, más allá de su número de habitantes. En la figura 4 se ha representado, en el eje de abscisas, la superficie artificial de cada una de las ciudades mayores de 10.000 habitantes, separándolas por tipos en el eje de ordenadas. Como era esperable, la dimensión de la superficie artificial guarda una relación general de proporcionalidad con la cantidad de población, siendo los tipos de ciudades más pobladas los que tienen mayor superficie artificial. Sin embargo, esta relación no se cumple cuando, en vez de observar los valores medios de los grupos de ciudades, se comparan ciudades individualmente. Por ejemplo, en el grupo de las grandes ciudades, Zaragoza con $121 \mathrm{Km}^{2}$ tiene una superficie artificial mucho mayor que Barcelona $\left(80 \mathrm{Km}^{2}\right)$, Valencia $\left(49 \mathrm{Km}^{2}\right)$ y Sevilla $\left(74 \mathrm{Km}^{2}\right)$, aunque tiene menos población $(933.000,114.000$ y 20.000 habitantes, respectivamente).

No obstante, sí resulta interesante conocer el rango de valores de la superficie artificial en que se mueve cada uno de los tipos. Así, las ciudades intermedias se sitúan entre los 12,6 $\mathrm{Km}^{2}$ de Badalona y los 70,1 $\mathrm{Km}^{2}$ de Murcia, con una media de $36,9 \mathrm{Km}^{2}$ de superficies artificiales. Las ciudades medias, de 50.000 a 200.000 habitantes, oscilan entre los 4,3 $\mathrm{Km}^{2}$ de Santa Coloma de Gramanet y los 48,4 Km² de Badajoz, con una media de $16,2 \mathrm{Km}^{2}$. Por último, para las pequeńas ciudades de menos de 50.000 habitantes, la media se sitúa en $5,1 \mathrm{Km}^{2}$, pero con grandes diferencias entre subtipos, pues para ciudades mayores a 30.000 habitantes la media es de $8 \mathrm{Km}^{2}$ y para las menores es de $4,5 \mathrm{Km}^{2}$.

De manera global, se puede indicar que la superficie artificial media de cada tipo es alrededor del doble del tipo precedente menos poblado, salvo en el escalón de las grandes metrópolis, en que la proporción pasa a ser mucho mayor. Esta relación justifica la utilidad de considerar tipos de ciudades según su población para estudiar sus características espaciales.

Por otra parte, los valores de la superficie artificial aportan información sobre el grado de transformación del territorio propio de cada tipo. Como se observa en el cuadro 1, para las ciudades pequeñas la superficie delimitada como artificial corresponde únicamente a un $4,3 \%$ de la suma de los términos municipales. Se trata, pues, de tipos de ciudades en los que la alteración del territorio derivada de la concentración de población es mucho menor que en las grandes ciudades, donde la superficie artificial es el 32,9\% de los términos. Si además se considera que la superficie media de los términos también aumenta cuando aumenta la población $\left(54,6 \mathrm{Km}^{2}\right.$ en municipios de menos de $10.000 \mathrm{hab}$., $118 \mathrm{Km}^{2}$ en pequeńas ciudades, $167,4 \mathrm{Km}^{2}$ en ciudades medias, $278,4 \mathrm{Km}^{2}$ en ciudades intermedias y 391,2 $\mathrm{Km}^{2}$ en grandes ciudades), queda de manifiesto que según se incrementa la población aumenta la presión que ejercen las superficies artificiales sobre el territorio. 
FIGURA 4 Extensión de superficies artificiales según ClC 2006 (en $\mathrm{Km}^{2}$ ) por tipos de ciudades. Con línea discontinua, valores medios; y en gráfico box-plot, valores mínimo, percentil 15\%, mediana, percentil $85 \%$ y máximo

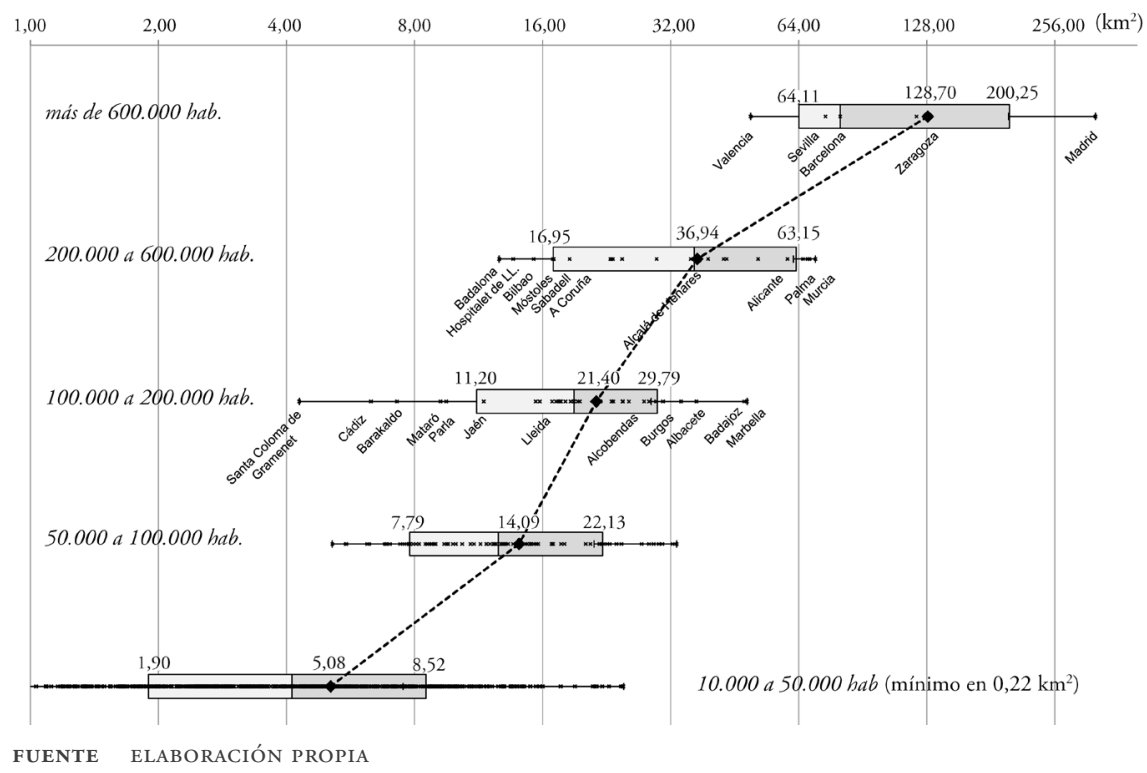

\section{Densidad de población}

La relación entre la cantidad de población y la superficie artificial representa la concentración de personas en el área, siendo utilizada en muchas ocasiones como indicador de la intensidad de actividades y funciones humanas en los entornos urbanos. En los distintos tipos de ciudades considerados en este estudio se observa que la densidad es mayor según aumenta la población del municipio (véase figura 5).

El conjunto de pequeñas ciudades de menos de 50.000 habitantes tiene una densidad media de 37,7 hab./ha, casi tres veces inferior a los 108,13 hab./ha de las cinco grandes ciudades de más de 600.000 habitantes. En este conjunto de pequeńas urbes se encuentran los valores extremos de densidad de todo el conjunto de ciudades, correspondientes a situaciones o modelos urbanos muy particulares. Con los valores mínimos de densidad podemos encontrar municipios como Puentes de García Rodríguez en La Coruña, 4,51 hab./ha, o Yaiza en Lanzarote, 6,43 hab./ ha. En el caso del término gallego, la inclusión de una antigua mina a cielo abierto dentro de las superficies artificiales es la causa de la baja densidad, mientras que en el caso canario, esta se debe a la delimitación como artificial del área de Playa Blanca, y especialmente de unos terrenos conocidos como El Rubicón, en los que en los años setenta se hicieron trabajos de medición para un complejo hotelero que dejaron su rastro en el terreno. 
Figura 5 | Densidad de población (en hab./ha) de los distintos tipos de ciudades. Con línea discontinua, valores medios; y en gráfico box plot, valores mínimos, percentil 15\%, mediana, percentil $85 \%$ y máximo

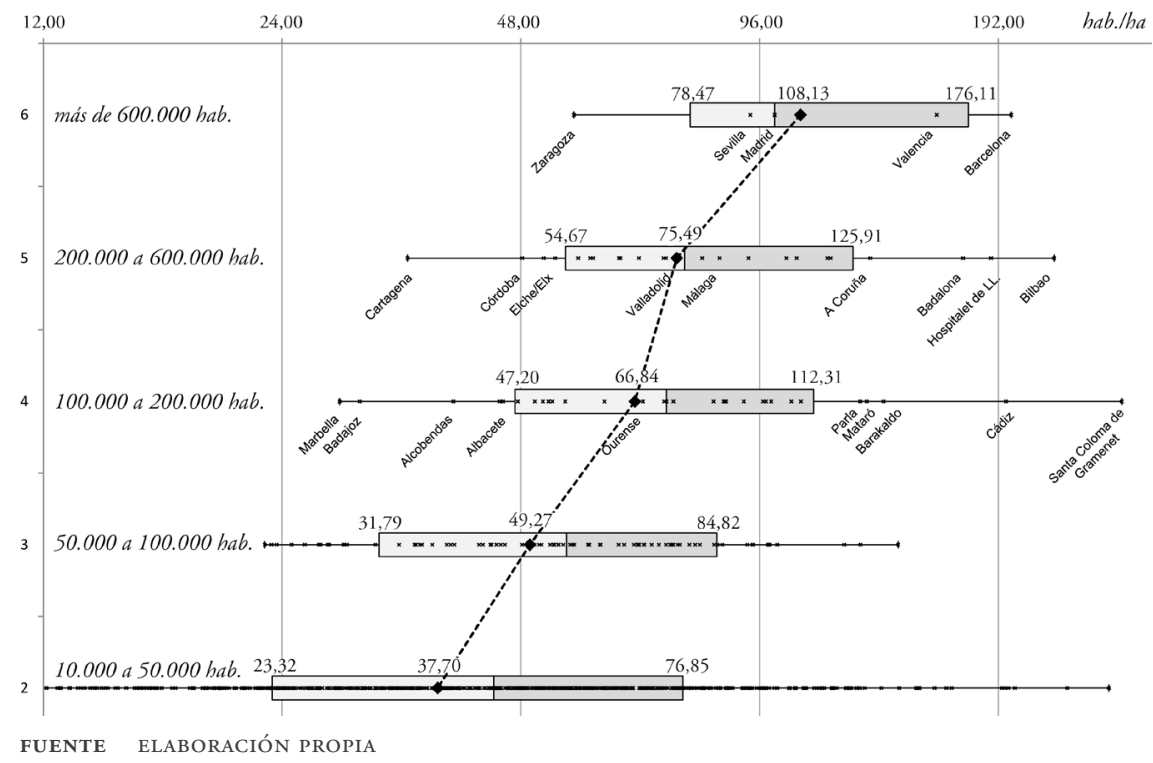

Si los valores mínimos de densidad corresponden a términos con áreas importantes de suelo transformado artificialmente, pero sin uso residencial, los valores máximos se deben a municipios con uso casi exclusivamente residencial, enclavados en áreas urbanas que abarcan varios municipios. Estos son los casos, por ejemplo, de Mislata en Valencia, 264,77 hab./ha, y Pasaia en Guipuzkoa, 234,55 hab./ha.

Una vez introducidos los casos correspondientes al tipo de situaciones urbanas presentes en los términos con densidades extremas, es interesante reducir el rango de densidad de este tipo a aquellos valores que comprenden el $70 \%$ de los casos, descartando los valores máximos y mínimos. Bajo estas circunstancias, en las ciudades pequeñas los valores quedan comprendidos entre los 24,4 y los 80,1 hab./ha.

Para el caso de ciudades medias, los valores oscilan entre los 22,81 y los 274,92 hab./ha, y entre los 35,44 y los 96,72 hab./ha en el rango del 70\% de los casos, siendo la media de 55,93 hab./ha. Entre las ciudades de más de 100.000 habitantes, los valores por debajo de los 30 hab./ha corresponden a municipios con fuerte presencia de urbanizaciones residenciales de baja densidad, tanto situados en el interior (Toledo, 27,42 hab./ha) como sobre todo costeros (Orihuela o Marbella), generalmente asociadas estas urbanizaciones a campos de golf. Para poblaciones menores a 100.000 habitantes, a las situaciones anteriores se les añade la presencia de grandes áreas de usos industriales. Los casos con densidad de población por encima de los 100 hab./ha se corresponden con términos de áreas metropolitanas (Santa Coloma de Gramanet, 274,92 hab./ha), en los que casi toda su superficie es ocupada por áreas residenciales, al igual que para las pequeńas ciudades y ciudades en que la orografía condiciona el crecimiento (como Cádiz, 196,33 hab./ha). 
FIGURA 6 | Mapa de municipios mayores de 10.000 habitantes según densidad de población

\section{Densidad}

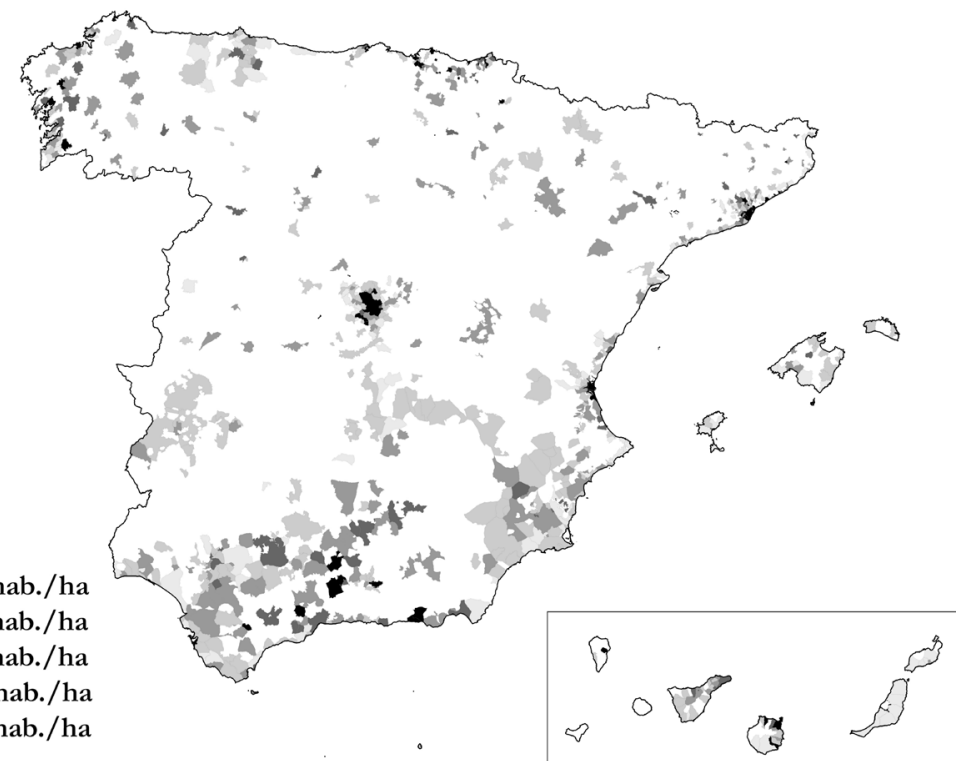

FUENTE ELABORACIÓN PROPIA

En el más reducido conjunto de ciudades intermedias, el 70\% de los casos se sitúa en un rango entre los 54,67 y los 125,91 hab./ha, que aumenta hasta los 34,54 y 225,86 hab./ha, incluyendo los 24 municipios de este tipo. La densidad de población media se encuentra en un valor ya bastante alto: 75,49 hab./ha. Únicamente Cartagena presenta un valor anómalamente inferior (34,54 hab./ha), debido a la presencia de grandes áreas industriales (Escombreras), zonas de minería (cerca de La Unión) y núcleos costeros de baja densidad (en el Mar Menor). Las situaciones que generan densidades por encima de los valores habituales son las mismas que para el tipo de ciudades medias, destacando Bilbao (225,86 hab./ha), L'Hospitalet de Llobregat (188,03 hab./ha) y Badalona (173,36 hab./ha).

Las grandes ciudades forman un conjunto tan reducido que el valor medio (108,13 hab./ha) no podría calificarse de representativo. De hecho, hasta los valores individuales de cada una están fuertemente influenciados por la división administrativa del territorio, dado que la ciudad funcional se extiende sobre varios municipios. Esto es especialmente visible en Valencia y Barcelona, cuyas altas densidades (160,6 y 199,38 hab./ha, respectivamente) se deben a que los usos no residenciales han sido desplazados a las zonas periféricas del conjunto metropolitano, pertenecientes a otros términos municipales. Sin embargo, Zaragoza es casi el ejemplo contrario: con poca fragmentación administrativa, su densidad es relativamente baja (55,99 hab./ ha), dado que soporta sobre su propia área todas las infraestructuras propias de las grandes urbes. Sería, pues, necesario un análisis conjunto de las áreas metropolitanas para este conjunto. 


\section{Compacidad}

Si se observaba que tanto la extensión artificial como la densidad de población se incrementaban para los tipos de ciudades más poblados, la compacidad mantiene un valor medio en torno al $30 \%$ y un rango -descartados los casos extremos- entre el $18 \%$ y el $48 \%$ para todos los casos estudiados. Al no apreciarse relación entre los tipos de ciudades por su población y su compacidad, se acude a hacer una distinción por criterios geográficos (véase figura 7). Atendiendo a los valores empíricos de compacidad de las superficies artificiales, se diferencian las ciudades por compacidad baja (menos del $25 \%$ ), media (25-35\%), alta (35-45\%) y muy alta (más del $45 \%)$.

FIGURA 7 | Mapa de municipios mayores de 10.000 habitantes según compacidad

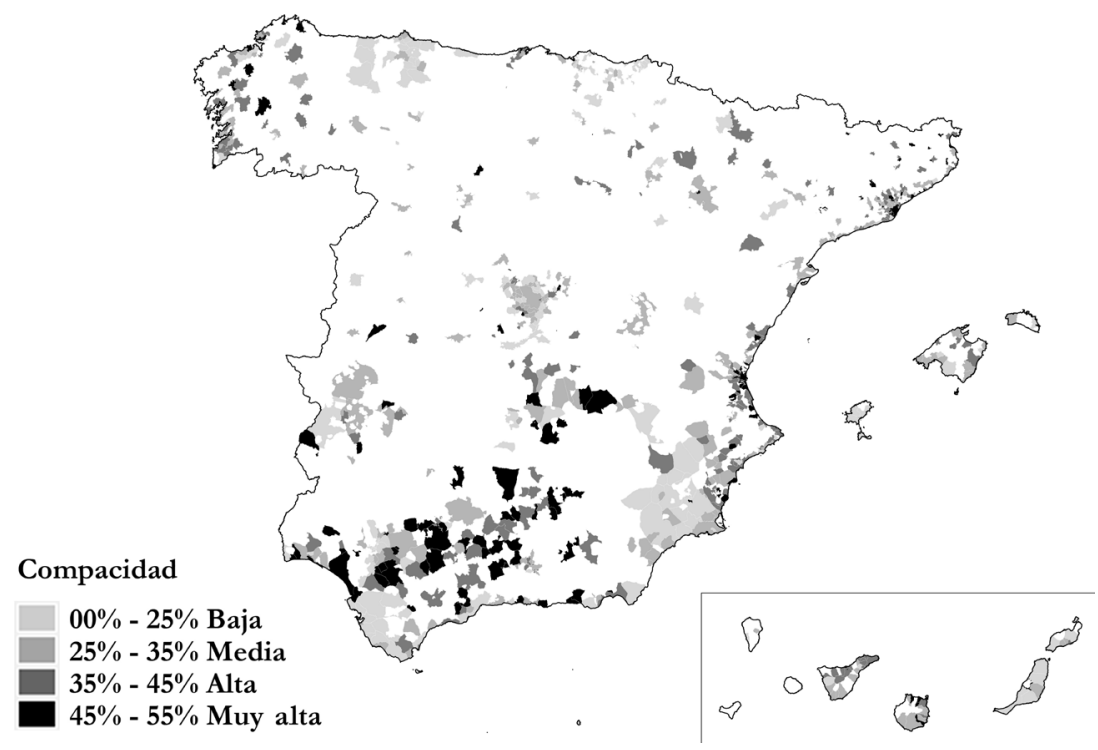

FUENTE ELABORACIÓN PROPIA

Las ciudades de baja compacidad se localizan principalmente en Asturias, País Vasco, el interior de Murcia, Cádiz y la corona más exterior del área metropolitana de Madrid. Como ciudades de compacidad media se encuentran, en primer lugar, algunas capitales interiores: Zaragoza, Cuenca, Lleida, Cáceres, Mérida o incluso Madrid y sus municipios más cercanos. De este mismo tipo se encuentran también algunos ejemplos en la costa gallega, la mediterránea de Andalucía, Cataluña y Murcia, y en el interior de la comunidad valenciana.

Las ciudades de alta compacidad son más características de todo el valle del Guadalquivir, el litoral valenciano, el área metropolitana de Barcelona y las rías gallegas. En estas mismas áreas, algunas ciudades alcanzan los valores definidos como de muy alta compacidad. Deben seńalarse entre estos casos, por su relevancia, los términos de Valencia, Barcelona y otros pertenecientes al núcleo central de estas áreas metropolitanas. 
Citymate. Diagrama de indicadores de la compacidad y la densidad de las ciudades Aunque los valores de compacidad y densidad resultan interesantes por sí mismos para conocer características espaciales de las ciudades, combinados es como ofrecen una información más completa. Para analizar conjuntamente estos valores se ha empleado una adaptación del diagrama Spacemate que realizaran Bergahuser y Haupt (2009), al que, siguiendo la nomenclatura, se ha denominado Citymate. La principal modificación respecto al Spacemate radica en que se emplea la densidad de población en vez de la densidad de la superficie edificada. En el eje de ordenadas se sitúa el valor de la densidad de población y en el de abscisas el valor de la compacidad.

Dado que ambos indicadores son índices referidos a la unidad de superficie, la distorsión derivada del diferente tamaño de las ciudades se elimina y puede utilizarse el diagrama para encontrar similitudes entre distintos casos. Aquellos que tengan características espaciales similares quedarán ubicados en zonas próximas del diagrama. Representando los distintos tipos de ciudades según su población en este diagrama, podemos ver cuáles son sus características espaciales y encontrar similitudes y diferencias (véase figura 8).

En el conjunto de grandes ciudades, se observa la gran distancia existente entre aquellas de compacidad media o baja (Zaragoza, Madrid y Sevilla) y las muy compactas (Barcelona y Valencia). Es destacable también el fuerte aumento de la densidad que supone el incremento de la compacidad en este tipo de ciudades. La consideración de las distintas áreas metropolitanas en conjunto ofrecería una comparación más precisa, pero aun así se intuye una diferenciación clara del modelo de ciudad compacta mediterránea.

En el conjunto de ciudades intermedias se observa también la relación directa entre densidad y compacidad, aunque en este caso el incremento es menor. Las densidades de los casos con compacidades muy altas, más del 45\% del suelo ocupado, se sitúan por encima de los 115 hab./ha. Para casos con compacidades entre el $35 \%$ y el $45 \%$ del suelo, el rango de la densidad tiene un mínimo de 55 y un máximo de 110 hab./ha, con la excepción de Badalona, que se eleva hasta los 170 hab./ha. Las ciudades de baja compacidad, entre $25 \%$ y $35 \%$, tienen un rango de densidades entre 30 y 110 hab./ha, de nuevo con una excepción muy significativa, como es Bilbao, que alcanza casi los 230 hab./ha. Por último, las bajas compacidades se caracterizan por valores de densidad entre los 50 y los 90 hab./ha.

El conjunto de ciudades medias ha sido dividido en dos subconjuntos, al apreciarse que para las ciudades entre 100.000 y 200.000 habitantes los valores de densidad eran sensiblemente mayores que para las ciudades entre 50.000 y 100.000 habitantes. En el subconjunto de ciudades de mayor tamańo, los tejidos con alta compacidad únicamente superan los 110 hab./ha en el caso de Cádiz, donde se alcanzan casi 200 hab./ha, mientras que las densidades mínimas están en torno a los 60 hab./ha, salvo el caso de Marbella, muy ligado al turismo de costa, donde es menor. En lo que respecta a las densidades medias, los límites de densidad son ligeramente inferiores, de 40 a 100 hab./ha. Se trata de rangos bastante estrechos, que indican una fuerte similitud de las características espaciales. Por el contrario, para las ciudades de baja densidad, inferior al 25\%, las características son menos homogéneas, con densidades que oscilan entre los 30 y los 140 hab./ha. 
Por su parte, en el subconjunto de ciudades menores, entre 50.000 y 100.000 habitantes, se aprecia un cambio en la relación entre densidad y compacidad, siendo mucho menos pronunciado el incremento de la primera en los casos más compactos. Las ciudades con tejidos de compacidad alta varían su densidad desde los 40 hasta los $100 \mathrm{hab} . / \mathrm{ha}$, mientras que las de compacidad media se mueven entre los $30 \mathrm{y}$ los 80 hab./ha. Por último, en la mayoría de las ciudades de compacidad baja las densidades oscilan entre los 20 y los 50 hab./ha. El último de los conjuntos, el de las ciudades menores de 50.000 habitantes, mantiene características espaciales similares. En general, los valores mínimos de densidad están en torno a los 10 hab./ ha, con el grueso de los casos situado por debajo de 70 hab./ha.

FIGURA 8 Diagrama Citymate de compacidad y densidad de las ciudades españolas y líneas de tendencia para cada uno de los tamaños de población. Figuran únicamente los nombres de las ciudades mayores de 200.000 habitantes

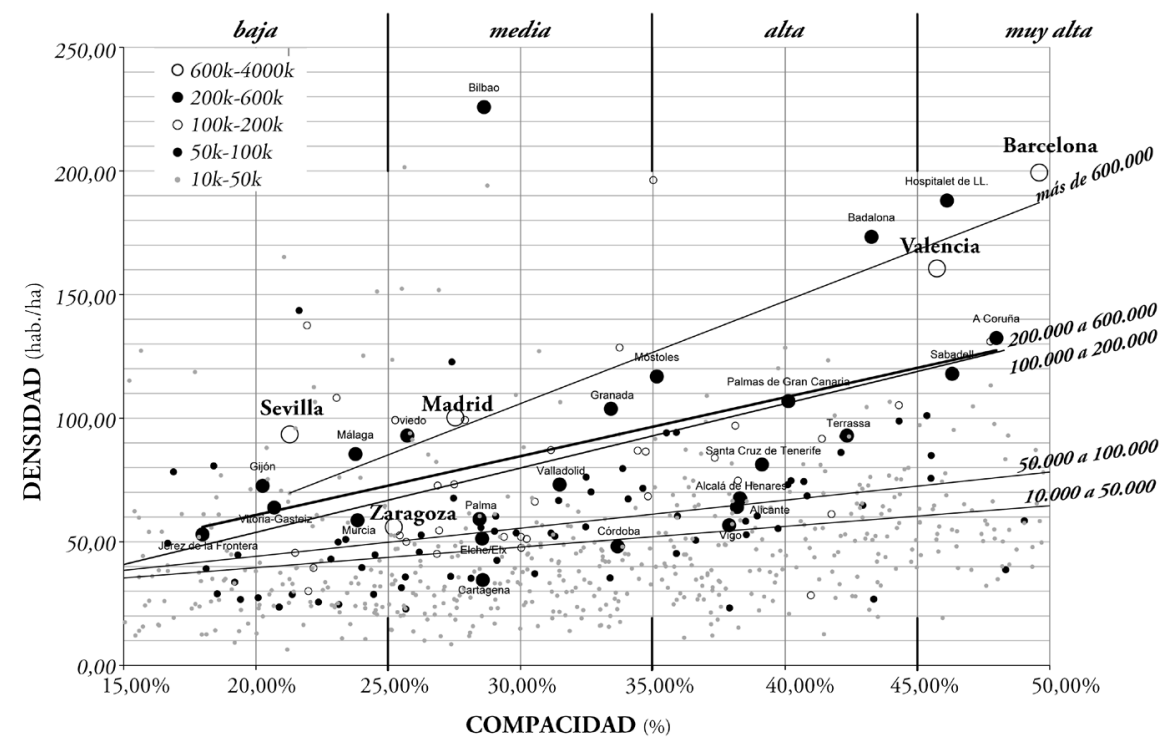

FUENTE ELABORACIÓN PROPIA

$\mathrm{Al}$ margen de la tendencia general, en estos últimos dos grupos — ciudades entre 10.000 y 100.000 habitantes - existe un número reducido de casos (considerando la amplitud del grupo) de compacidad media-baja, pero con valores de densidad notablemente superiores, llegando a alcanzar los 150 hab./ha. En su mayoría corresponden a pequeñas ciudades industriales del País Vasco, en las que altas edificaciones se disponen sobre una orografía que no permite una ocupación mayor del suelo o a núcleos de la periferia norte de Pamplona. 


\section{Conclusiones}

A partir del estudio expuesto se puede enunciar una serie de conclusiones. Las primeras están relacionadas con la validez de la metodología de cálculo empleada, que ofrece nuevas posibilidades a futuros estudios que tengan en cuenta las variables de densidad y compacidad.

- Se ha comprobado la utilidad de las fuentes de datos existentes, especialmente aquellas referidas a coberturas del suelo (CLC y SIOSE), para el cálculo de los dos valores considerados, densidad y compacidad. La continuidad de estos proyectos permitiría un estudio de la evolución de la morfología de las ciudades que resultaría, sin duda, de interés.

- Además, se ha observado que estas fuentes presentan ciertas restricciones en su uso, especialmente en lo referido a la unidad mínima cartografiable de CLC, que se ha demostrado inadecuada para la medición de las superficies artificiales en los municipios de menor tamaño. Estas limitaciones sirven de observación para futuros trabajos, pero, además, deberían llevar a considerar modificaciones en la metodología de elaboración de las bases de datos de coberturas del suelo.

Los resultados obtenidos han permitido delimitar los rangos de compacidad y densidad de las ciudades españolas. Si bien el hecho de trabajar con un número elevado de ciudades, 755 de muy distintas características, ha llevado a evitar el empleo de más variables, sin duda sí deberían estar presentes en estudios con un número de casos más reducido o con otro tipo de objetivos. A la vez, los resultados individuales de cada tipo de ciudad permiten un análisis más detallado, que no ha sido incluido en este artículo por cuestiones de espacio.

- Se han establecido los rangos de las superficies artificiales y de la densidad de población sobre ellas para cada tipo de ciudad, rangos que son crecientes según aumenta el número de habitantes. Los valores medios de superficies artificiales de cada tipo indican las fuertes diferencias existentes en la extensión de las áreas urbanas: de los $5 \mathrm{Km}^{2}$ de media para las ciudades de menor población (10.00050.000 habitantes) hasta los $129 \mathrm{Km}^{2}$ de las grandes ciudades. Las densidades de población medias también varían de manera notable entre tipos, desde los $37,7 \mathrm{hab} . /$ ha de las pequeñas ciudades a los 108,13 hab./ha de las mayores. De esta forma se constata que las ciudades más pobladas requieren de mayor superficie artificial y presentan una concentración de habitantes superior.

- No se ha observado que existan diferencias entre los rangos de compacidad de los distintos tipos de ciudad según tamaño. En todos ellos los valores de la ocupación del suelo variaban entre $18 \%$ y $48 \%$, si descartamos los casos extremos (percentil 15\% y 85\%).

- El estudio conjunto de la compacidad y densidad de población en el diagrama Citymate ha permitido observar relaciones entre ambas variables. Para las ciudades más pobladas, por encima de 100.000 habitantes, los modelos más com- 
pactos implican un aumento de la densidad de población. Sin embargo, para ciudades de menos población esta relación es mucho más débil.

- Se ha comprobado que el Citymate tiende a agrupar ciudades con características espaciales similares en el diagrama, lo que ha llevado a centrar la atención en la forma de la ciudad. Aunque se han apuntado puntualmente características de los distintos modelos (ciudades litorales con fuerte presencia de áreas residenciales de baja densidad, ciudades "dormitorio" en grandes áreas metropolitanas, ciudades con grandes bolsas de suelo destinadas a uso industrial, etcétera), se ha evitado establecer una clasificación tipológica, pues esta debe responder a los objetivos para los que vaya a ser empleada posteriormente.

\section{Referencias bibliográficas}

Agencia de Ecología Urbana de Barcelona. (2007). Plan especial de indicadores de sostenibilidad ambiental de la actividad urbanistica de Sevilla. Avance. Barcelona: Ayuntamiento de Sevilla/Agència d'Ecologia Urbana de Barcelona. En http://www.sevilla.org/ urbanismo/plan_indicadores/0-indice.pdf

Agencia de Ecología Urbana de Barcelona. (2009). Plan de indicadores de sostenibilidad urbana de Vitoria-Gasteiz. Vitoria-Gasteiz: Ayuntamiento de Vitoria-Gasteiz/Agència d'Ecologia Urbana de Barcelona. En http://bit.ly/21zYovq

Aguado, M. D. (Coord.). (2010). Capitales \& Ciudades+100. Información estadística de las ciudades españolas 2010. Madrid: Centro de Publicaciones, Secretaría General Técnica, Ministerio de Fomento. En http://bit.ly/1shNTkA

Aguado, M. D. (Coord.). (2013). Áreas urbanas+50. Información estadística de las Grandes Áreas Urbanas españolas 2012. Madrid: Centro de Publicaciones, Secretaría General Técnica, Ministerio de Fomento. En http://bit.ly/221xBIE

Angel, S., Parent, J., Civco, D. L. \& Blei, A. M. (2011). Making room for a planet of cities. Policy Focus Report. Cambridge, Ma: Lincoln Institute of Land Policy. En http://bit. ly/1OpoHwG

Azcárate Luxán, M. V., Cocero Matesanz, D., Fernández Fernández, A., García Lázaro, F. J., Muguruza Cañas, C. \& Santos Preciado, J. M. (2012). El proceso de urbanización dispersa de las metrópolis españolas, en el contexto del desarrollo urbano europeo. Uned. Espacio, Tiempo y Forma, Serie VI (3), 13-26. En http://dx.doi.org/10.5944/ etfvi.3.2010.2611

Bellet, C. \& Llop, J. Ma. (1999). Ciudades intermedias y urbanización mundial. Lleida: Ajuntament de Lleida/Unión Internacional de Arquitectos (UIA), Unesco, Ministerio de Asuntos Exteriores de España. En http://www.unesco.org/most/ciudades.pdf

Berghauser, M., (2011). Measuring urban form. Atlantis, 22(2), 16-19.

Berghauser, M. \& Haupt, P. (2009). Space, density and urban form. Rotterdam: NAi Uitgevers (тU Delft Institutional Repository). En http://bit.ly/27jFxZK

Blanco, J. (2004). La emergencia de las nuevas ciudades en la era global. Gijón: Trea. 
Bossard, M., Feranec, J. \& Otahel, J. (2000). CORINE land cover technical guide - Addendum 2000. Copenhague: European Environment Agency. En http://www.eea.europa.eu/ publications/tech40add/page001.html.

Boyko, C. T. \& Cooper, R. (2011). Clarifying and re-conceptualising density. Progress in Planning, 76 (1), 1-61. En http://dx.doi.org/10.1016/j.progress.2011.07.001

Burton, E. (2000). The potential of the compact city for promoting social equity. En M. Jenks, K. Williams \& E. Burton (Eds.), Achieving sustainable urban form (pp. 19-29). Londres: E\&FN Spon.

Büttner, G., Feranec, J. \& Jaffrain, G. (2006). CORINE land cover nomenclature illustrated guide Addendum 2006. Copenhague: European Environment Agency. En https://www.epa. ie/pubs/data/corinedata/CLC2006\%20Final\%20Report.pdf

Capel, H. (1975). Capitalismo y morfología urbana en España. Barcelona: Los Libros de la Frontera.

European Comission (2011). Eurostat regional yearbook 2011. Luxemburgo: Publications Office of the European Union.

European Environment Agency. (2006). Urban sprawl in Europe. The ignored challenge. EEA report (Vol. 10). Luxemburgo. En http://eprints.uwe.ac.uk/10295/

Fariña Tojo, J. \& Naredo, J. M. (Dirs.). 2010. (2010). Libro Blanco de la Sostenibilidad en el Planeamiento Urbanistico Español. Madrid: Ministerio de la Vivienda, Gobierno de España.

Font, A. (2007). Morfologías metropolitanas contemporáneas de la baja densidad. En F. Indovina (Coord.), La ciudad de baja densidad. Lógicas, gestión y contención (pp. $97-$ 107). Barcelona: Diputació de Barcelona.

Ganau, J. \& Vilagrasa, J. (2003). Ciudades medias en España: posición en la red urbana y procesos urbanos recientes. En H. Capel (Coord.), Ciudades, arquitectura y espacio urbano (pp. 37-73). El Ejido: Caja Rural Intermediterránea. Cajamar. En http://bit. ly/1T9bLgL

García, F. M. (2012). Evolución de los usos en relación con los parámetros morfológicos de densidad y compacidad (Una muestra en Puente de Vallecas, Madrid). Territorios en Formación, (2), 37-50. En http://polired.upm.es/index.php/territoriosenformacion/ article/view/1772

Goerlich, F. J. (2013). Áreas rurales y coberturas del suelo. Documentos de Trabajo (No. 2/2013) (p. 53) Bilbao: Fundación BBva. En http://www.fbbva.es/TLFU/dat/ DT_02_2013\%20_web.pdf

Goerlich, F. J. \& Cantarino, I. (2012). Una grid de densidad de población para España. Madrid. Fundación BBVA.

Goerlich, F. J. \& Cantarino, I. (2013). Zonas de morfología urbana: coberturas del suelo y demografía. Madrid: Fundación вBVA.

Gropious, W. (1930). Bauhausbauten Dessau. Múnich: A. Langen.

Hillier, B. \& Hanson, J. (1984). The social logic of space. Cambridge, MA: Cambridge University Press. 
Hurtado, C. (2013). La dimensión urbana de la ocupación del suelo a través del Corine Land Cover y el Mapa de Usos y Coberturas Vegetales de Andalucía. En XXIII Congreso de Geógrafos Españoles (AGE). Espacios insulares y de frontera, una visión geográfica (pp. 383392), Palma (Mallorca), Universitat de les Illes Balears, 23 al 25 de octubre de 2013. En http://bit.ly/1R11PDt

Jenks, M., Burton, E. \& Williams, K. (Eds.). (1996). The compact city. A sustainable urban form? Nueva York: Routledge.

Kickert, C. C., Berghauser Pont, M. \& Nefs, M. (2014). Surveying density, urban characteristics, and development capacity of station areas in the Delta Metropolis. Environment and Planning B: Planning and Design, 41 (1), 69-92. En http://doi.org/10.1068/b39020

López de Lucio, R. (2007). Construir ciudad en la periferia: criterios de diseño para áreas residenciales sostenibles. Madrid: Mairea.

March, L. \& Martin, L. (1972). Urban spaces and structures. Londres: Cambridge University Press.

Marcus, L. (2005). Plot Syntax: A configurational approach to urban diversity. Paper, Fifth International Space Syntax Symposium, Delft, 13-17 de junio 2005. tu Delft: Techne Press. En http://spacesyntax.tudelft.nl/media/Papers/076.pdf

Masnavi, M. (2000). The new millennium and the new urban paradigm: The compact city in practice. En K. Williams, E. Burton \& M. Jenks (Eds.), Achieving sustainable urban form (pp. 64-73). Londres: E\&FN Spon.

Moya González, L. (1983). Barrios de Promoción Oficial. Madrid: 1939-1976. Madrid: Colegio Oficial de Arquitectos de Madrid-

Navarro, J. R. \& Ortuño, A. (2011). Aproximación a la génesis de la contribución de la densidad en la noción de "ciudad compacta". EURE, 37 (112), 23-41. En http:// dx.doi.org/10.4067/S0250-71612011000300002

Owens, S. (1986). Energy, planning and urban form. Londres: Pion.

Steadman, P. (2014). Density and built form: Integrating 'Spacemate' with the work of Martin and March. Environment and Planning B: Planning and Design 2, 41 (2), 341-358. doi: $10.1068 / \mathrm{b} 39141$

Terán Troyano, F. de. (1982). Planeamiento urbano en la España contemporánea (1900/1980). Madrid: Alianza.

Thinh, N. X., Arlt, G., Heber, B., Hennersdorf, J. \& Lehmann, I. (2002). Evaluation of urban land-use structures with a view to sustainable development. Environmental Impact Assessment Review, 22 (5), 475-492. En http://dx.doi.org/10.1016/S01959255(02)00023-9

Uytenhaak, R. (2008). Cities full of space. Qualities of density. Rotterdam: nai010 publishers.

Vinuesa, J. (1989). La población de las ciudades medias españolas. Urbanismo. Revista Oficial del Colegio de Arquitectos de Madrid (COAM), (6), 17-27.

Webster, C. (2010). Pricing accessibility: Urban morphology, design and missing markets. Progress in Planning, 73 (2), 77-111. En http://dx.doi.org/10.1016/j.progress.2010.01.001

Williams, K. (2000). Does intensifying cities make them more sustainable? En K. Williams, E. Burton \& M. Jenks (Eds.), Achieving sustainable urban form (pp. 30-45). Londres: E\&FN Spon. 
